\title{
$N 94-12296$
Direct numerical simulation of turbulent flow over a backward-facing step
}

\author{
By H. Le AND P. Moin
}

\section{Objectives}

The objectives of this study are: (a) to conduct a direct numerical simulation of turbulent backward facing step flow using inflow and outflow conditions and (b) to provide data in the form of Reynolds stress budgets for Reynolds averaged modeling. The report presents the basic statistical data and comparisons with the concurrent experiments of Jovic and Driver $(1991,1992)$ and budgets of turbulent kinetic energy.

\section{Accomplishments}

\subsection{Method}

The Navier-Stokes equations are discretized using a finite difference method on a staggered mesh. Uniform mesh spacing is applied in the streamwise $(x)$ and spanwise $(z)$ directions. In the vertical $(y)$ direction, non-uniform mesh is employed with mesh refinement at the wall and near the step. The fractional step method from Le and Moin (1990) is used for time advancement. The Navier-Stokes equations are first advanced using a second-order semi-implicit method without the pressure terms. The pressure is calculated by solving the Poisson equation, and the velocities are then corrected to satisfy the continuity equation.

The mean velocity profile obtained from a boundary layer simulation by Spalart (1986) is imposed at the inlet at $R e_{\theta}=667$. Random velocity fluctuations $u^{\prime}, v^{\prime}$, and $w^{\prime}$ are superimposed on this profile according to a variant of the method of Lee et al. (1992). The fluctuations are prescribed such that, at the inlet, the turbulence intensities and Reynolds shear stress of Spalart's data are also duplicated. A convective boundary condition (Pauley et al., 1988) is imposed at the exit.

The streamwise domain consists of an entry section of length $10 \mathrm{~h}$ prior to the step and a $20 h$ post-expansion section, where $h$ is the step height. The vertical dimensions before and after the expansion are $W_{1}=5 h$ and $W_{2}=6 h$ which give an expansion ratio $E R$ of 1.20 . The spanwise dimension is $4 h$ where periodic boundary conditions are imposed. The simulation uses $770 \times 194 \times 66$ grid points in the streamwise, wall normal, and spanwise directions, respectively. The Reynolds number, based on $h$ and the mean inlet free stream velocity $U_{0}$, is $R e_{h}=5100$. The computation uses 13 megawords of memory and requires approximately $55 \mathrm{CPU}$ seconds per time step on a single processor CRAY Y-MP at a rate of 186 mflops. Statistical quantities are averaged over the spanwise direction and time. About $1100 \mathrm{CPU}$ hours were required to obtain an adequate statistical sample. The total computational time corresponds to approximately 4.5 flow-through times. 


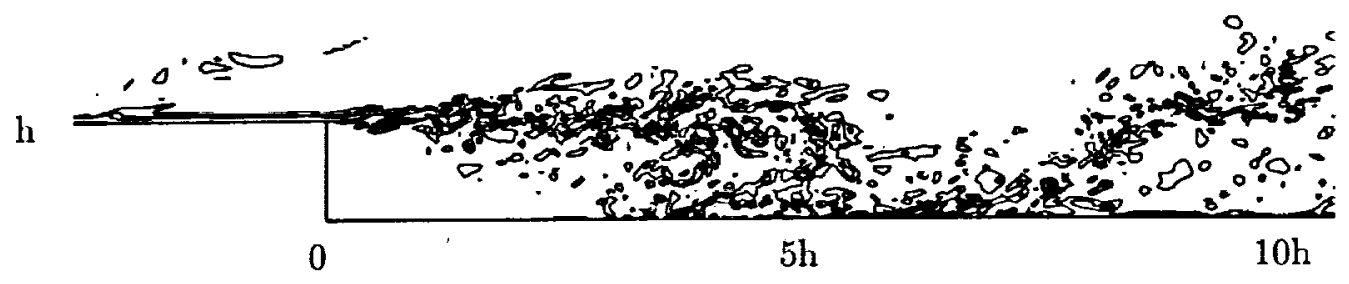

FIGURE 1. Instantaneous spanwise vorticity contours, $\omega_{z} ; \min =-7, \max =4.95$, increment $=1.3$.

\subsection{Results}

Figure 1 shows contour plots of the instantaneous spanwise vorticity $\omega_{z}$ on a typical vertical plane. The vorticity is normalized by $U_{0} / h$. A free-shear layer spreading from the step and interacting with the lower wall near the mean reattachment location, $x_{R}=6 h$, is discernible.

The basic statistical quantities are compared to results from concurrent experinents by Jovic and Driver. In 1991, they conducted a backward facing step experiment at $R e_{h}=6800$ and $E R=1.09$, herein referred as "JD1". However, the results indicated that these parameters are not sufficiently close to those used in simulations. Thus, Jovic and Driver in 1992 conducted a second experiment with $R e_{h}=4950$ and $E R=1.20$ ("JD2"). In the following sections, JD2 results will be used for comparison with numerical simulations.

\subsubsection{Reattachment length}

The instantaneous velocity fields indicate that the reattachment location oscillates in the streamwise direction and time and also varies in the spanwise direction. Several methods were used to determine the mean reattachment location, $x_{R}$ : (a) by the location at which the mean velocity $U=0$ at the first grid point away from the wall, (b) by the location of zero wall-shear stress $\left(\tau_{w}=0\right)$, and (c) by the location of the mean dividing streamline $(\psi=0)$. A pdf method was also used in which the mean reattachment point is indicated by the location of $50 \%$ forward flow fraction. The pdf method was also used experimentally by Westphal et al. (1984) and Adams et al. (1984). The results of the first three methods are within $0.1 \%$ of each other and about $4 \%$ from the pdf result. The calculated mean reattachment length is $6.0 h$ compared to $6.1 \mathrm{~h}$ measured in JD2 experiment.

\subsubsection{Pressure distribution}

The streamwise pressure coefficient at the step-wall is compared with the JD2 results in Fig. 2. The pressure coefficient is normalized by the mean inlet freestream velocity. The two symbols in Fig. 2 are for the two walls of the double-sided expansion in the JD2 experiment. The comparison shows an excellent agreement between computation and experiment. 


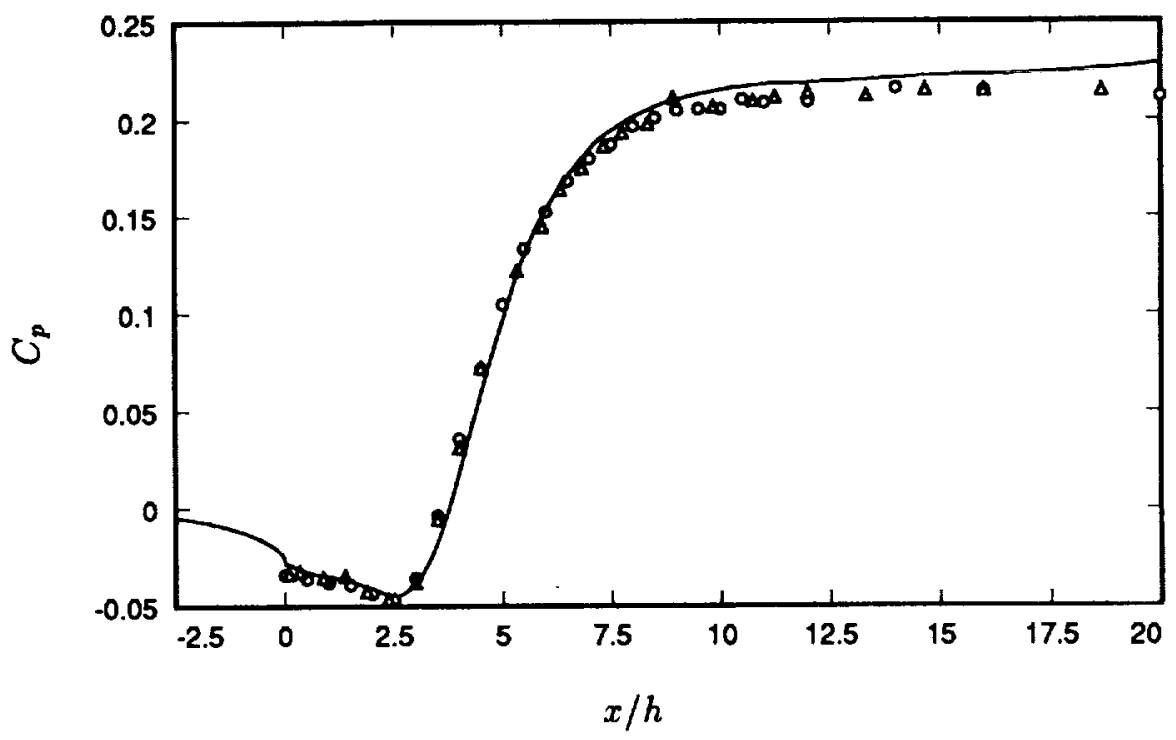

FIGURE 2. Step-Wall pressure coefficient as a function of $x$. - , computation; o , Jovic \& Driver (1992), bottom wall; $\triangle$, Jovic \& Driver (1992), top wall.

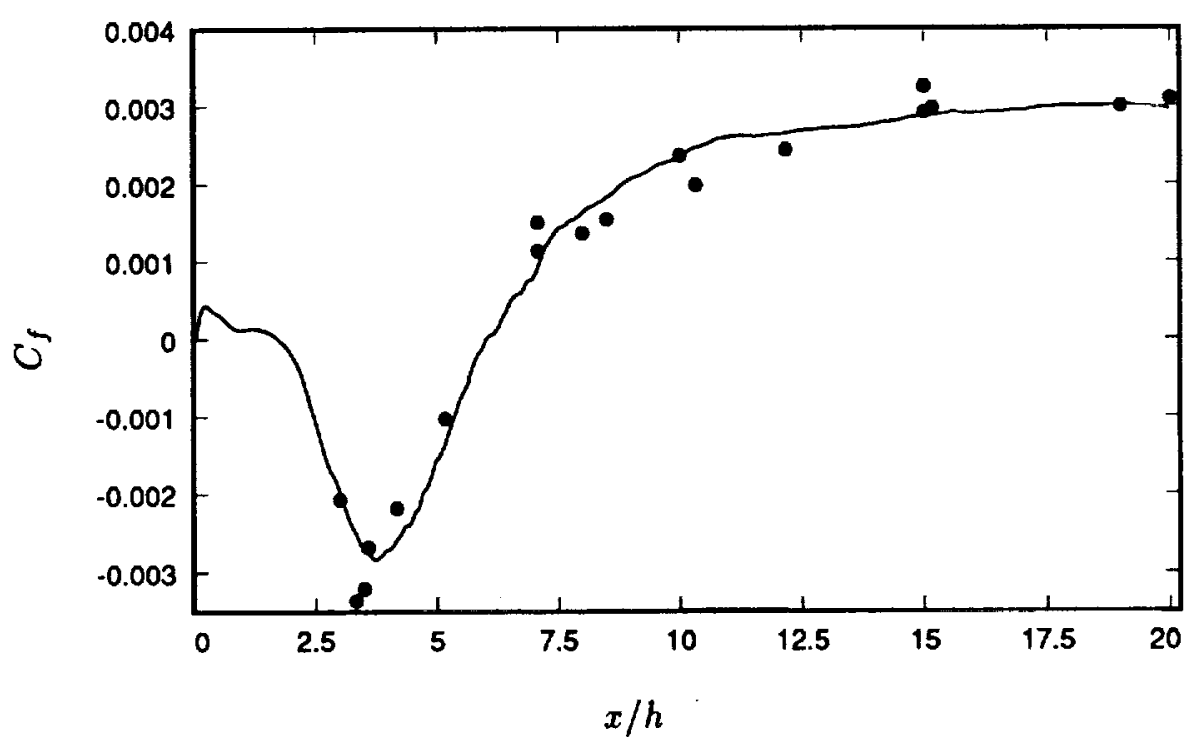

FIGURE 3. Step-wall coefficient of friction. - , computation; $\bullet$, Jovic \& Driver (1992). 


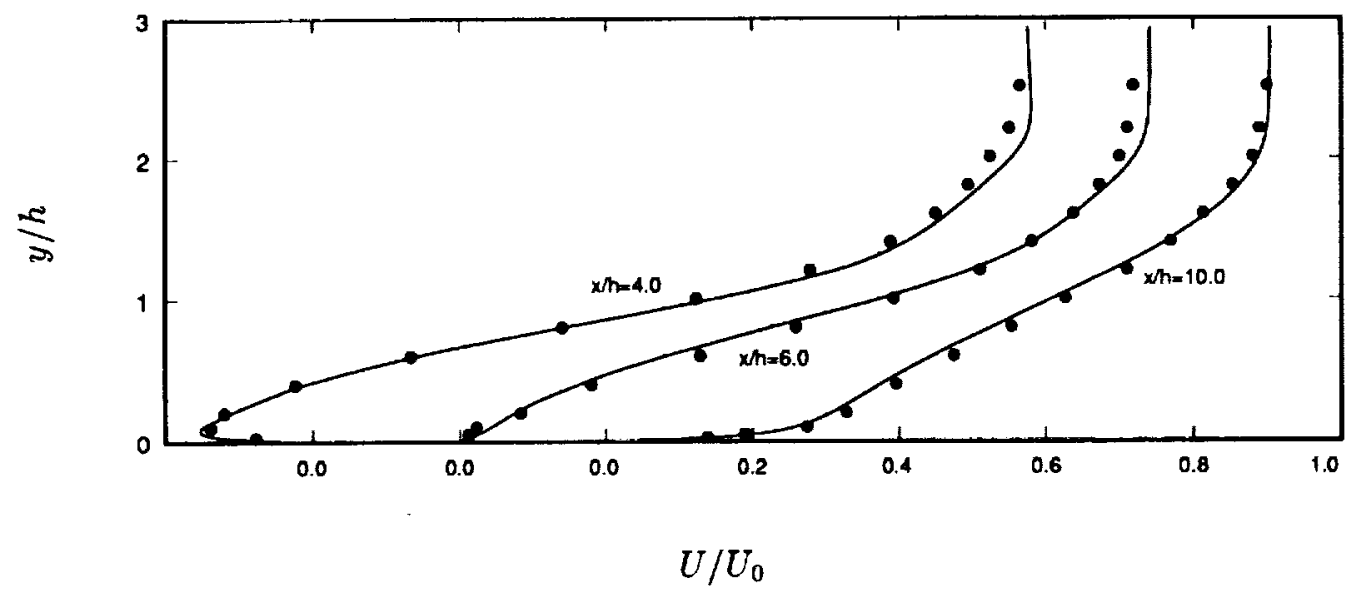

Figure 4. Mean velocity profiles. — , computation; • Jovic \& Driver (1992).

\subsubsection{Skin friction coefficient}

The skin friction coefficient $C_{f}$ is computed from the mean velocity profile and shown in Fig. 3. Also plotted are the $C_{f}$ data from the JD2 experiment. The large peak negative skin friction in the recirculation region $\left(\approx-3 \times 10^{-3}\right)$, seen in both computation and experiment, is nearly 3 times larger than previous experimental findings, e.g. Adams et al. (1984). Jovic and Driver (1991) also measured the skin friction in the reverse flow region for a wide range of Reynolds numbers. Their results show that the recirculation $C_{f}$ decreases with increasing Reynolds number. The peak negative $C_{f}$ reaches a value of approximately $-1.0 \times 10^{-3}$ as $R e_{h} \approx 40000$ which is the Reynolds number range used in many experiments. Thus the large skin friction in the current study is due to low Reynolds number effects.

\subsubsection{Mean velocity profiles}

Figure 4 shows the mean velocity profiles compared with JD2 experimental results at three $x$-locations: $x / h=4$ (recirculation region), $x / h=6$ (reattachment location), and $x / h=10$. Again, excellent agreement is obtained between computation and JD2 experiment.

The near-wall mean velocity profile in the recovery region at $x / h=19$ is compared to experimental data in Fig. 5. The shear velocity $u_{\tau}$ of the JD2 experiment is calculated from the skin friction coefficient of Fig. 3. Above $y^{+}=10$, both profiles show a noticeable shift below the log-law, indicating that the turbulent boundary layer has not fully recovered. Previous studies, however, reported a recovery of the log-law profile even as early as $x-x_{R}=6 h$, e.g. Westphal et al. (1984). The apparent discrepancy is attributed to the method of obtaining the wall-shear velocity $u_{\tau}$. In these experiments, the wall-shear velocity was calculated using the Clauser chart with the inherent assumption that the log-law of the zero-pressure gradient turbulent boundary layer is applicable. The result was lower values of $u_{\tau}$. 


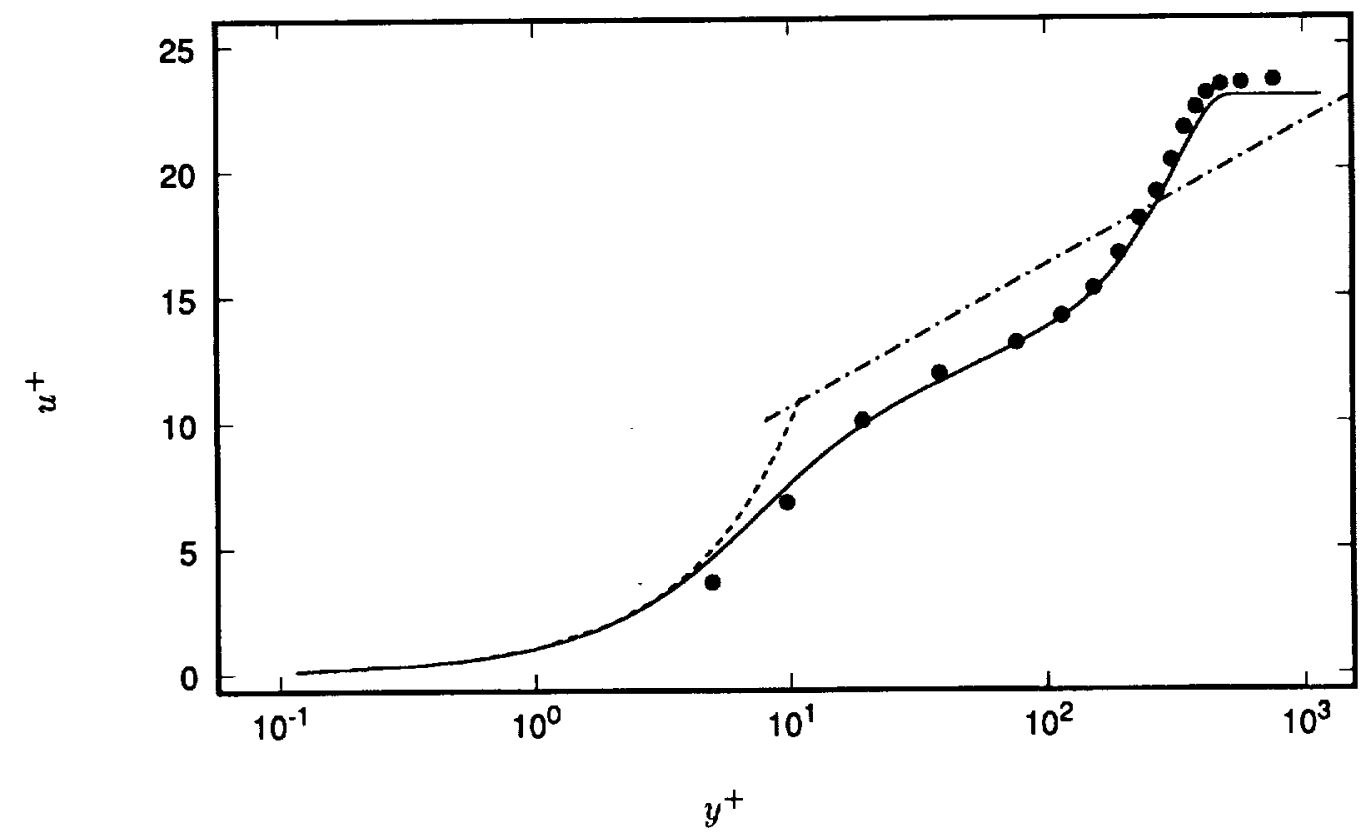

Figure 5. Mean velocity profile at $x / h=19 .-\cdots, u^{+}=y^{+} ;-\cdots$, $u^{+}=(1 / .41) \log \left(y^{+}\right)+5.0 ;-$, computation; $\bullet$, Jovic \& Driver (1992).

Direct $C_{f}$ measurements using a pulsed wall probe by Westphal et al. confirm that the correct shear velocity in this flow region is indeed higher than that predicted by the Clauser chart. In the present configuration, this discrepancy is as high as $17 \%$, i.e., $u_{\tau} / u_{\tau_{c}} \approx 1.17$, where the subscript $c$ denotes the value obtained using the Clauser chart. The deviation of the velocity profile from the log-law may be due to the effect of the strong streamwise adverse pressure gradient which is experienced by the flow following the sudden expansion (Nagano et al., 1991). It may also be due to non-equilibrium effects which are persistent after reattachment.

\subsubsection{Turbulence intensities and Reynolds stress}

The turbulence intensity and Reynolds shear stress profiles, normalized by the inlet free-stream velocity $U_{0}$, are shown in Figs. 6-8. They are compared with those from JD2 experiment data at streamwise locations, $x / h=4,6,10$. The overall agreement is good.

\subsubsection{Turbulent kinetic energy budget}

The terms of the turbulent kinetic energy transport equation are evaluated from the equation

$$
\frac{\partial q^{2}}{\partial t}=\underbrace{-U_{k} \overline{\left(u_{l}^{\prime} u_{l}^{\prime}\right)}}_{C_{k}} \underbrace{-2 \overline{u_{l}^{\prime} u_{k}^{\prime}} U_{l, k}}_{P_{k}} \underbrace{-\overline{\left(u_{l}^{\prime} u_{l}^{\prime} u_{k}^{\prime}\right)}}_{T_{k}} \underbrace{+\frac{1}{R e} \overline{\left(u_{l}^{\prime} u_{l}^{\prime}\right)}}_{D_{k}}, \underbrace{-\frac{2}{R e} \overline{u_{l, k}^{\prime} u_{l, k}^{\prime}}}_{\epsilon_{k}} \underbrace{-2 \overline{u_{l}^{\prime} p_{l, l}^{\prime}}}_{\Pi_{k}} .
$$




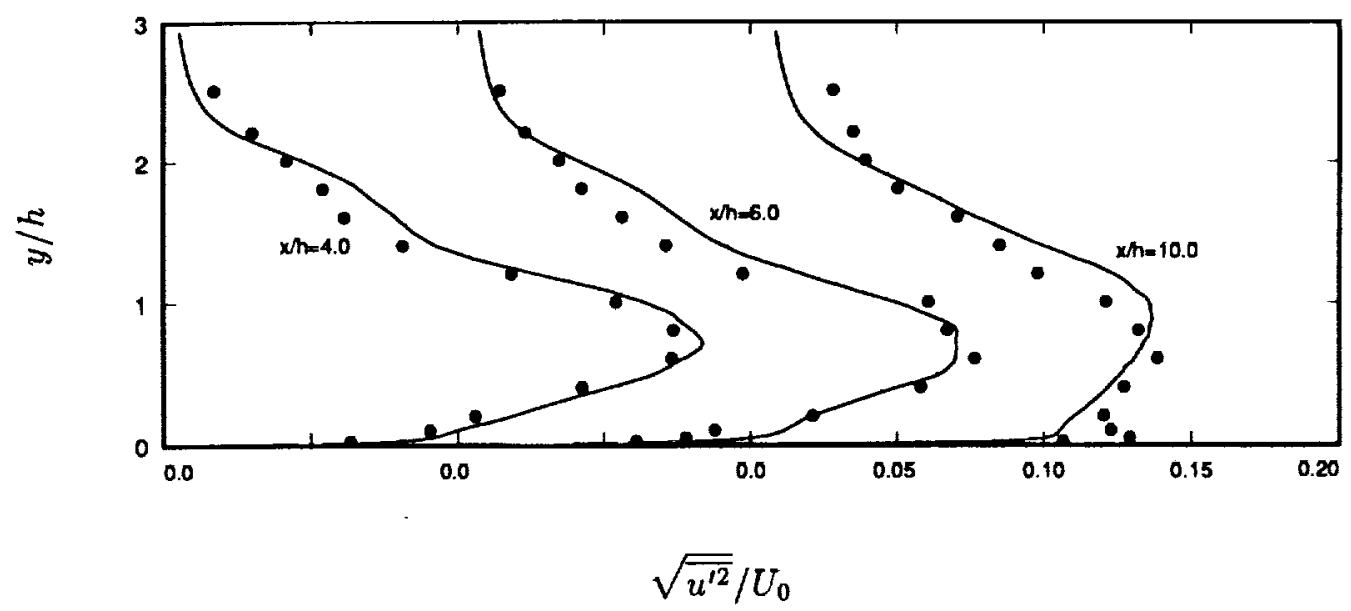

FIgURE 6. Turbulence intensity $\sqrt{\overline{u^{\prime 2}}}$, normalized to inlet mean velocity $U_{0}$. , computation; • , Jovic \& Driver (1992).

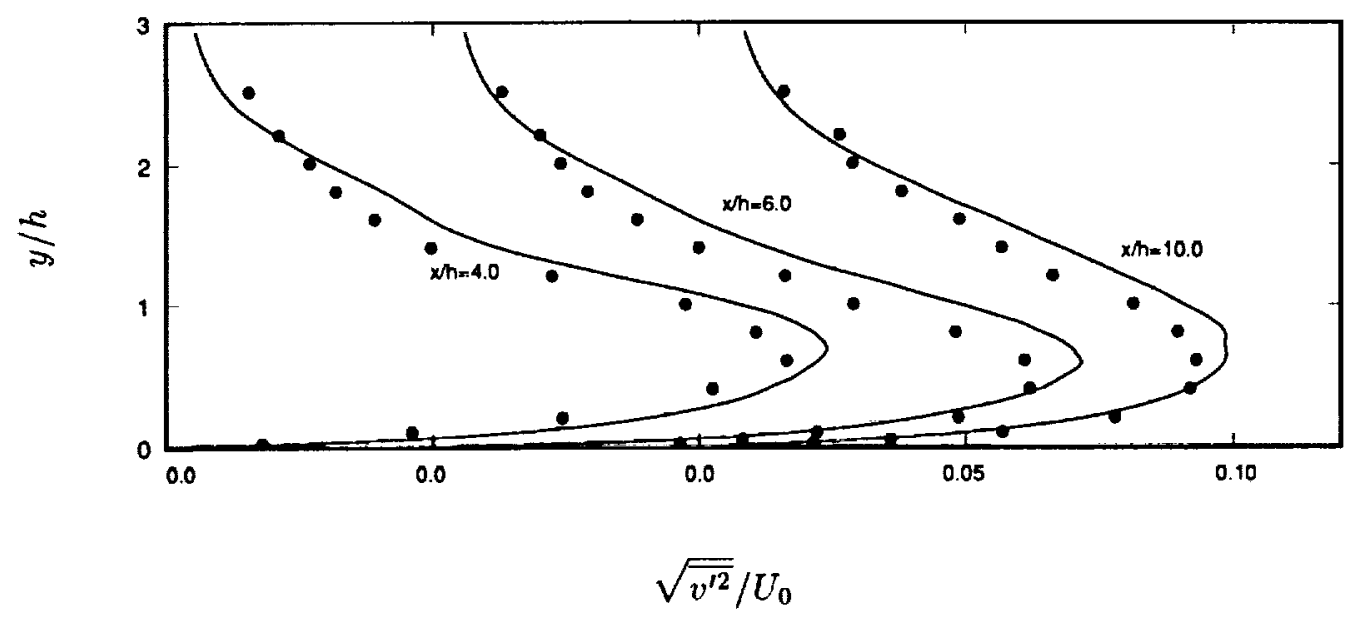

FIGURE 7. Turbulence intensity $\sqrt{\overline{v^{\prime 2}}}$, normalized to inlet mean velocity $U_{0}$. - computation; $\bullet$, Jovic \& Driver (1992). 


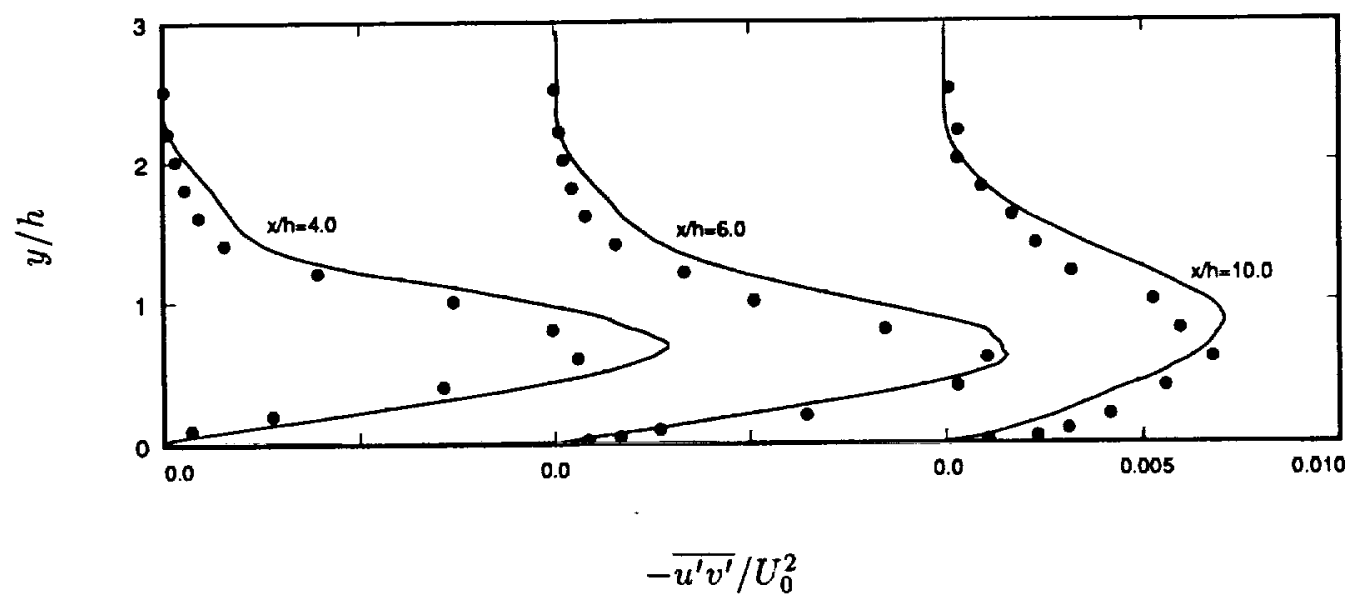

FIGURE 8. Reynolds shear stress $-\overline{u^{\prime} v^{\prime}}$, normalized to inlet mean velocity $U_{0}^{2}$. - computation; • , Jovic \& Driver (1992).

The terms on the right hand side are identified as follows:

$C_{k}=$ Convection,

$P_{k}=$ Production,

$T_{k}=$ Turbulence Diffusion,

$D_{k}=$ Viscous Diffusion,

$\epsilon_{k}=$ Viscous Dissipation,

$\Pi_{k}=$ Velocity Pressure-Gradient.

The budget for the turbulent kinetic energy, $\frac{q^{2}}{2}=\frac{1}{2}\left(\overline{u_{i}^{\prime} u_{i}^{\prime}}\right)$, is shown in Figs. 9 and 10 .

At two step heights before the separation, the energy budget (Figs. 9(a) and $10(a)$ ) is similar to that of a turbulent boundary layer (Spalart, 1988) although there is an enhancement of the viscous terms near the wall. The budget for the recirculation region is shown in Fig. 9(b). This energy budget is very similar to that of a plane mixing layer (Bradshaw and Ferriss, 1965). This budget also agrees qualitatively with the measurement by Chandrsuda and Bradshaw (1981) for a backward facing step flow. Both production $P_{k}$ and viscous dissipation $\epsilon_{k}$ have maximum values at the same point in the free-shear layer. The peak $\epsilon_{k}$ is approximately $60 \%$ of the production peak. The other $40 \%$ is balanced by the turbulence transport $T_{k}$. Far from the wall, $P_{k}$ is the only major gain term. $T_{k}$ is a consuming term for $0.3<y / h<1$, but a gain term outside of this range. A bove the step $(y / h>1), T_{k}$ is in balance with $C_{k}$, the turbulence convection by the mean flow. As one approaches the wall, the production becomes a consuming term because of the negative gradient of the mean reverse flow (Fig. 10(b)) although its magnitude is relatively small. Very close to the wall, the two viscous terms, $D_{k}$ and 

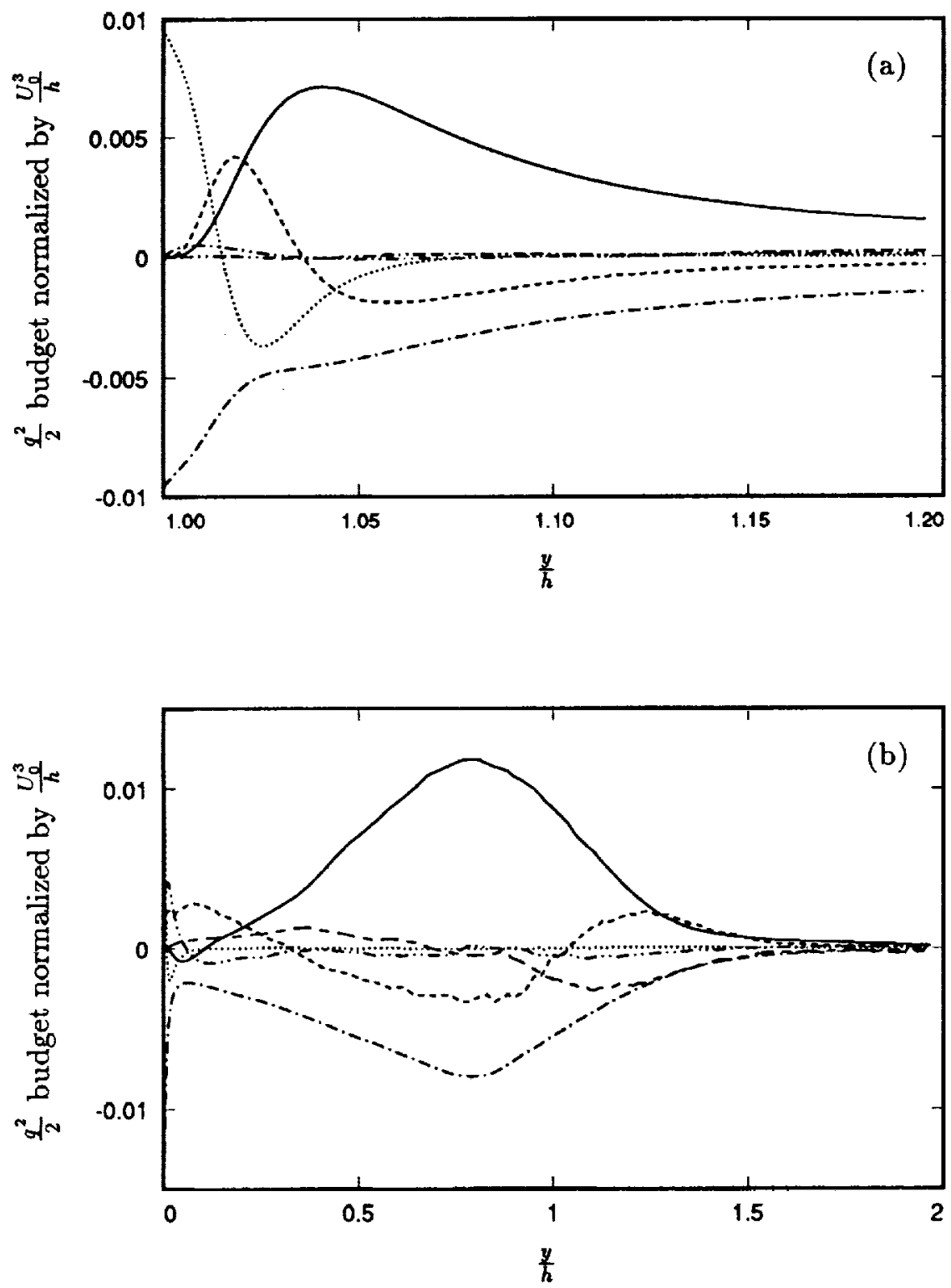

FIGURE 9. Budget terms for turbulent kinetic energy, $\frac{q^{2}}{2}=\frac{1}{2}\left(\overline{u_{i}^{\prime} u_{i}^{\prime}}\right)$, away from the wall, normalized by $U_{0}^{3} / h$. (a) $x / h=-2.0$; (b) $x / h=4$. - , production; ---- , turbulence transport; $\cdots \cdots . .$. , viscous diffusion; - - - , viscous dissipation; $-\cdots-$, velocity pressure-gradient; $-\cdots$, , convection. 

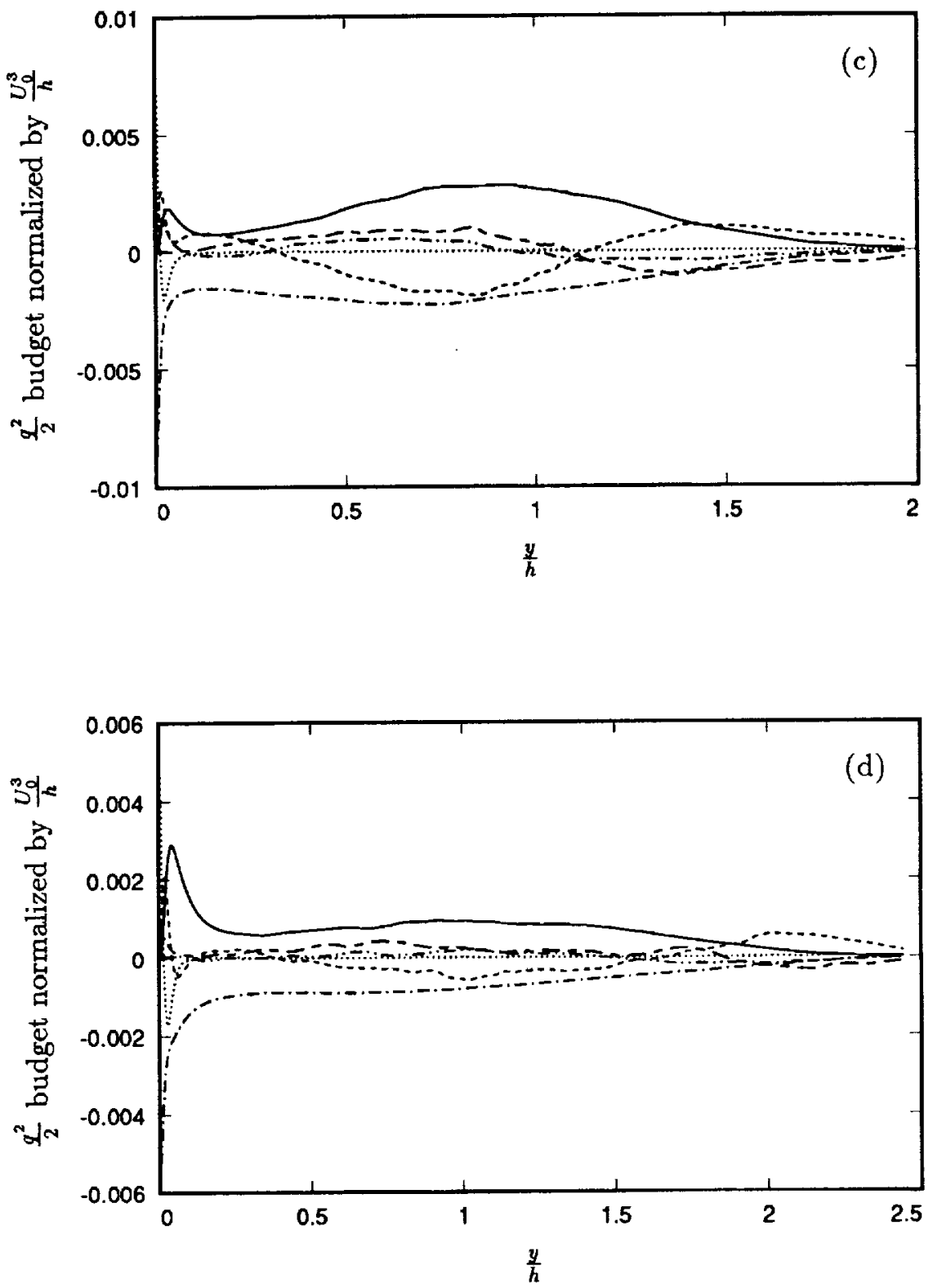

Figure 9. (cont.) Turbulent kinetic energy budget away from the wall, normalized by $U_{0}^{3} / h$. (c) $x / h=10$; (d) $x / h=18$. For caption see previous page. 

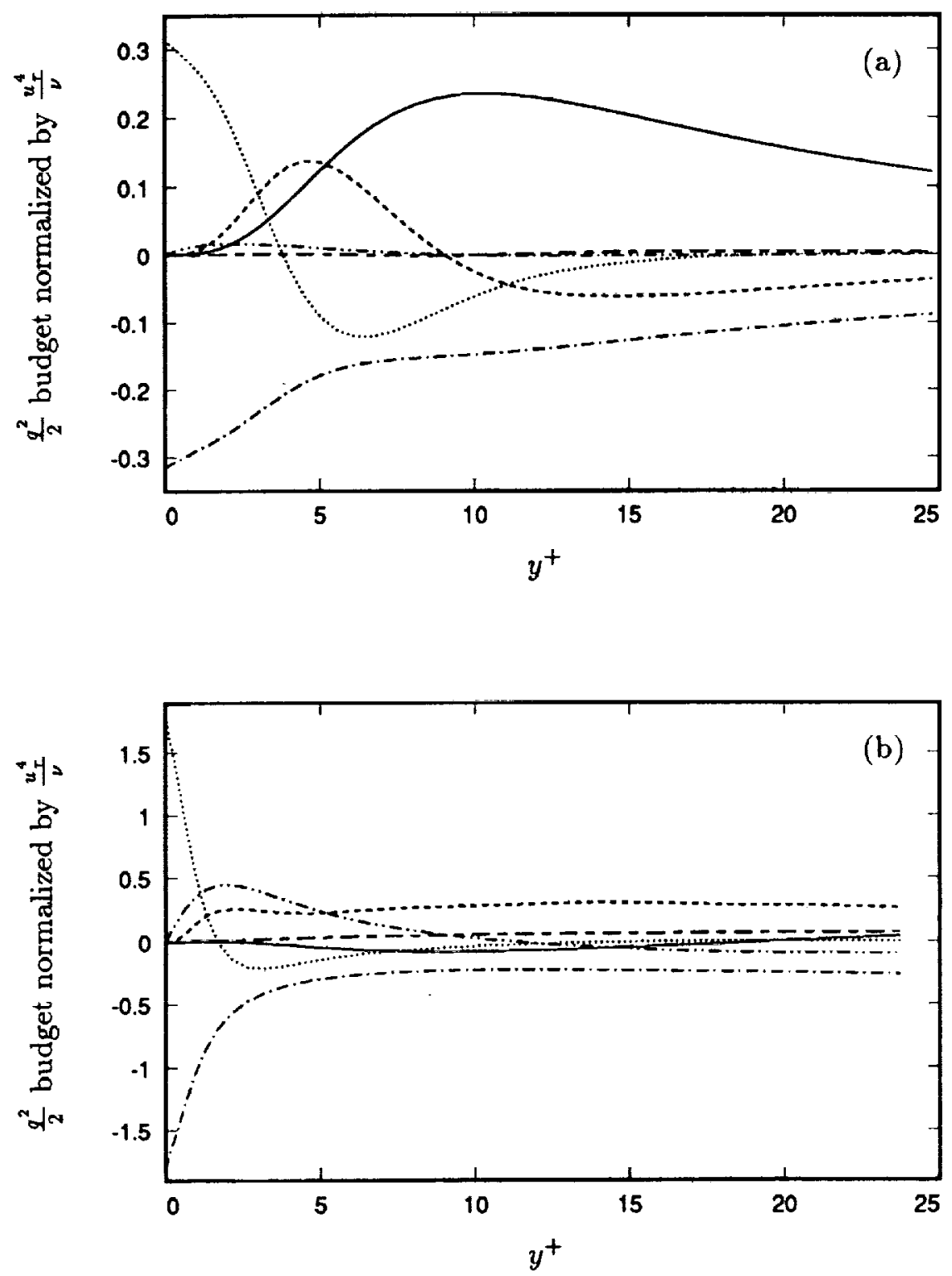

Figure 10. Budget terms for turbulent kinetic energy, $\frac{q^{2}}{2}=\frac{1}{2}\left(\overline{u_{i}^{\prime} u_{i}^{\prime}}\right)$, normalized by local $u_{\tau}^{4} / \nu$. (a) $x / h=-2.0$; (b) $x / h=4$. - , production;----, turbulence transport; $\cdots \cdots . .$. , viscous diffusion; - - , viscous dissipation; $-\cdots-$, velocity pressure-gradient; --- convection. 

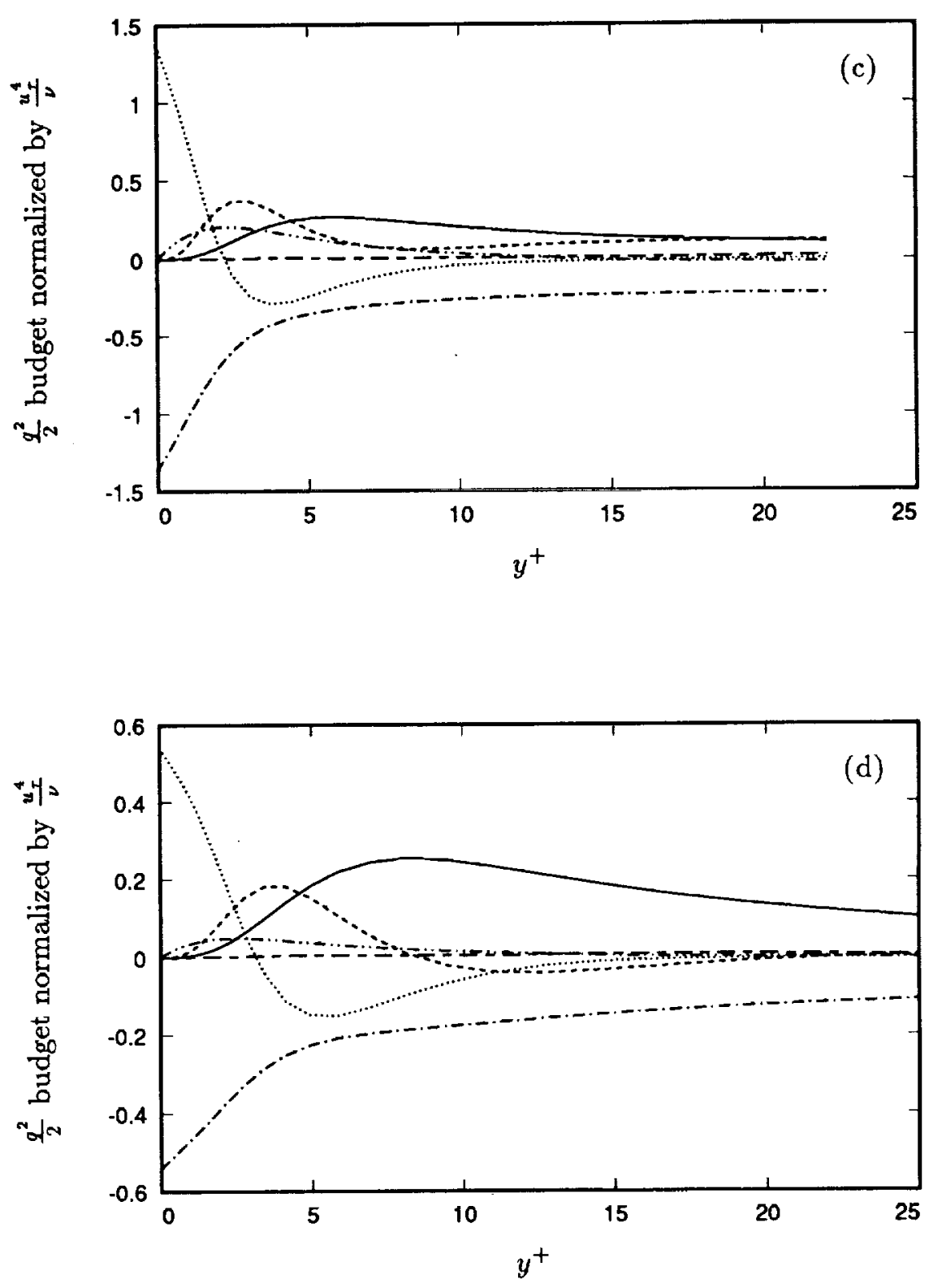

Figure 10. (cont.) Budget terms for turbulent kinetic energy near wall, normalized by $u_{\tau}^{4} / \nu$. (c) $x / h=10 ;$ (d) $x / h=18$. For caption see previous page. 
$\epsilon_{k}$, grow rapidly due the high intensities in all three directions (their value at the wall is about $40 \%$ higher than the peak production in the shear layer). The velocity pressure-gradient term becomes significant only at $y^{+}<8$ where it balances the viscous terms plus $T_{k}$. The profiles have similar shapes through the reattachment point.

All terms decay with $x$; however, the energy in the shear layer decays much faster than at the wall (the streamwise decay of the budget terms are not apparent in Fig. 10 because they are normalized with the local $u_{\tau}$ ). By $x / h=10$, the value of $D_{k}$ at the wall is about 3 times the peak production value. Near the flow exit, $y / h=18$, the turbulent kinetic energy budget resembles that of a boundary layer. However, the effects of the free-shear layer is still apparent, e.g., $T_{k}$ is still large at $y / h=1$ and the peak $\epsilon_{k}$ is only $\approx 85 \%$ of the peak $P_{k}$.

\section{Conclusions}

A direct numerical simulation of turbulent flow over a backward facing step at $R e_{h}=5100$ was successfully completed. There is good agreement in turbulence statistics deduced from simulations and concurrent experiments of Jovic and Driver. Of interest are two observations not previously reported for the backward-facing step flow: (a) at the relatively low Reynolds number considered, large negative skin friction is seen in the recirculation region; the peak $\left|C_{f}\right|$ is about three times the value reported at high Reynolds numbers; (b) the velocity profiles in the recovery region fall below the universal log-law.

A large data base from this recently completed simulation has been archived. It contains up to third-order statistics at all locations in the recirculation, reattachment, and recovery zones and the budgets of all components of the Reynolds stress tensor.

\section{Acknowledgements}

This research was funded by the National Science Foundation (NSF) and the Center for Turbulence Research (CTR). We are grateful to Dr. J. Kim for very insightful discussions throughout the course of this study. The authors are also specially grateful to Drs. D. Driver and S. Jovic for their collaboration and performing the concurrent experiments.

\section{REFERENCES}

Adams, E. W., Johnston, J. P. \& Eaton, J. K. 1984 Experiments on the structure of turbulent reattaching flow, Report MD-43, Department of Mechanical Engineering, Stanford University.

Bradshaw, P. \& Ferriss, D. H. 1965 The spectral energy balance in a turbulent mixing layer, AERO Report 1144, National Physical Laboratory, England, 6.

Chandrsuda, C. \& BrAdSHAW, P. 1981 Turbulence structure of a reattaching mixing layer. $J$. Fluids Mech. 110, 171-194.

Jovic, S. \& Driver, D. 1991, 1992 private communication. 
LE, H. \& MoIN, P. 1991 An improvement of fractional step methods for the incompressible Navier-Stokes equations. J. Comp. Physics. 92, 369-379.

LEE, S., LELE, S. K. \& MoIN, P. 1992 Simulation of spatially evolving turbulence and the applicability of Taylor's hypothesis in compressible flow. Phys. of Fluids A. 4, 1521-1530.

Nagano, Y., TAGaWA, M. \& Tsuji, T. 1991 Effects of adverse pressure gradients on mean flows and turbulence statistics in a boundary layer, Proceedings of the Eighth Symposium on Turbulent Shear Flows, Technical Univ. of Munich, 2-31-2-3-6.

Pauley, L. L., MoIn, P. \& Reynolds, W. C. 1988 A numerical study of unsteady laminar boundary layer separation, Report TF-34, Department of Mechanical Engineering, Stanford University.

SPALART, P. R. 1988 Direct simulation of a turbulent boundary layer up to $R_{\theta}=$ 1410. J. Fluids Mech. 187, 61-98.

WestPhal, R. V., Johnston, J. P. \& Eaton, J. K. 1984 Experimental study of flow reattachment in a single-sided sudden expansion, Report MD-41, Department of Mechanical Engineering, Stanford University. 
